\title{
Benign Hepatic Tumors: From Incidental Imaging Finding to Clinical Management
}

\author{
Fadi Abu Baker ${ }^{1,2, *}$, Abdel-Rauf Zeina ${ }^{3,4}$, Saif Abu Mouch ${ }^{1,2}$, Amir Mari ${ }^{1,2}$ \\ 'Gastroenterology and Hepatology Institute, Hillel Yaffe Medical Center, Hadera, Israel \\ ${ }^{2}$ Ruth and Rappaport Faculty of Medicine, Haifa, Israel \\ ${ }^{3}$ Department of Radiology, Hillel Yaffe Medical Center, Hadera, Israel \\ ${ }^{4}$ Faculty of Medicine, Technion-Israel Institute of Technology, Haifa, Israel
}

Benign hepatic tumors (BHTs) are commonly detected as incidental finding mainly due to the frequent utilization of imaging modalities, including ultrasound (US), computed tomography (CT), and magnetic resonance imaging (MRI). Rigorous clinical evaluation, with a particular focus on chronic liver disease (CLD) or risk factors for CLD, medication history, physical examination for signs of CLD, blood tests, and a review of past liver radiology constitute the initial steps in the evaluation of a new liver lesion. Further, contrast-enhanced imaging using US, CT and MRI, can be used depending on the clinical scenario and their availability. The contrast-enhanced MRI provides detailed tissue assessment while avoiding exposure to radiations, although it is scarcely available and expensive. While the liver tissue-specific protocols ensure precise diagnosis, a biopsy is recommended in selected or doubtful cases. Further, most BHTs, such as hemangiomas, are harmless and do not require special management or followup, the hepatic adenomas and large or atypical cases of focal nodular hyperplasia are clinically relevant and require management/follow-up. In such cases, it is favorable to have a multidisciplinary team approach, which includes hepatologist, radiologist, hepatobiliary surgeon, and pathologist. This review aims to elaborate the current understanding of BHTs, and provide a practical guidance for primary care and practitioners of family and internal medicine for the disease evaluation and management.

Keywords: Benign Hepatomas; Diagnosis; Focal Nodular Hyperplasia; Hepatic Adenoma

Received: November 12, 2018, Revised: January 8, 2019, Accepted: January 17, 2019

${ }^{*}$ Corresponding Author: Fadi Abu Baker https://orcid.org/0000-0001-5428-7180

Tel: +972-47744480, Fax: +972-47744408, E-mail: fadia@hy.health.gov.il 


\section{INTRODUCTION}

With an extensive use of radiologic modalities, a considerable number of liver lesions are being diagnosed in routine clinical practice. SmithBindman et al. ${ }^{1)}$ observed a massive increase in the usage of radiologic modalities between 1996 and 2010, which has contributed to the increased rate of diagnosis of liver lesions. Most of these lesions are incidental findings in patients being evaluated for other conditions, such as kidney stones, epigastric pain, and other non-specific complaints. Hepatic lesions may origin from all kind of liver cells including hepatocytes, mesenchymal as well as cholangiocellular line, and a reliable detection and characterization of liver lesions is critical for optimal patient management. Primary care providers are not uncommonly the first practitioners to encounter patients with imaging findings of liver lesions and thus should have a basic knowledge as well as tools to deal with these findings in order to avoid any undesirable consequences. Fortunately, the majority of liver lesions arising in non-cirrhotic liver are benign. Cysts, hepatic hemangiomas ( $\mathrm{HH}$ ), focal nodular hyperplasias (FNH), and hepatic adenomas (HA) are among the most commonly encountered benign lesions. Despite the benign nature, HAs have been associated with a significant risk of hemorrhage and malignant transformation and thus need to be followed more closely than other benign tumors. Moreover, atypical cases of FNH or HH may have serious consequences that mandate specific management strategies. Hepatocellular carcinomas (HCC), and to a lesser extent intrahepatic cholangiocarcinomas, represent the most common primary liver malignancies and are diagnosed primarily in the setting of cirrhosis or chronic liver disease, while the most commonly encountered malignant lesions in non-cirrhotic liver are metastases.

An outstanding development of new imaging techniques has taken place during the last decade. Contrast-enhanced imaging is the most sensitive diagnostic method, and facilitates the accurate diagnosis of majority of the lesions based on specific liver imaging protocols. ${ }^{2)}$ Still, differentiation of masses may be complex, and biopsy or resection for histological diagnosis sometimes becomes necessary.

In the current study we reviewed recent literature and society guidelines concerned with BHT evaluation and management. The current review outlines the major differential diagnosis of benign hepatic masses in adults and discusses an algorithmic approach of manage- ment for primary care practitioners.

\section{HEPATIC HEMANGIOMAS}

HHs are the most prevalent form of benign hepatic tumors (BHTs). They are asymptomatic and discovered incidentally during imaging, and the prevalence observed is up to $20 \%$ in autopsy specimens and $5 \%$ in radiological modalities. ${ }^{4,5)} \mathrm{HHs}$ are frequent in women aged 30 40 years, with a female to male ratio of 5:1. ${ }^{6}$ Though HHs are usually less than $4 \mathrm{~cm}$ in size and are solitary, giant and multiple HHs have also been reported. While HHs are hepatic vascular malformations with a poorly understood aetiology, their origin either as a congenital malformation or as an aberrant vascular growth, remains to be determined. ${ }^{7)}$ Although the use of oral contraceptives has been linked with $\mathrm{HH}$, the association could not be established in the case-controlled study by Gemer et al. ${ }^{8)}$ Moreover, majority of the HHs are asymptomatic and clinically irrelevant. However, approximately $11 \%$ of the HHs, especially those of large sizes, may cause symptoms due to pressure on adjacent organs, which may be manifested as pain in the right upper quadrant, dyspepsia, nausea, and vomiting. Additionally, HHs may be complicated by bleeding or consumptive coagulopathy with thrombocytopenia and generalized bleeding, a condition known as Kasabach-Merritt syndrome. ${ }^{9)}$

HHs may be diagnosed using US, CT, or MRI (Table 1), where the $\mathrm{HH}$ typically appear as homogenous, hyperechoic lesions generally smaller than $3 \mathrm{~cm}$ in size with a well-defined rim and a few intra-nodular vessels in US. The contrast-enhanced imaging shows peripheral enhancement in the arterial phase, followed by centripetal enhancement in the delayed phases (Figure 1) ${ }^{10}{ }^{10}$ According to the guidelines of the European Association for the Study of the Liver (EASL) published in 2016, US images depicting typical characteristics of a HH is sufficient for diagnosis, if the lesion has size $<3 \mathrm{~cm}$ with no chronic liver disease. ${ }^{11)}$ Furthermore, MRI is the preferred modality as it has the highest specificity and sensitivity for identifying HHs. However, due to their benign nature, no imaging follow-up is required for typical HHs. ${ }^{11,12)}$

As per the guidelines of the EASL and American Association for the Study of Liver Diseases (AASLD), oral contraceptives and hormonal therapy are not contraindicated in these patients. However, though the

Table 1. Imaging characteristics of FNH and $\mathrm{HH}$

\begin{tabular}{|c|c|c|c|}
\hline $\begin{array}{l}\text { Hepatic benign } \\
\text { tumor }\end{array}$ & Ultrasound & CT & Magnetic resonance imaging \\
\hline FNH & $\begin{array}{l}\text { Generally lobulated isoechoic or slightly } \\
\text { hypoechoic } \\
\text { Central hyperechoic scar can be visualized in } \\
\text { some cases } \\
\text { Doppler: a central feeding artery with a stellate or } \\
\text { spoke wheel may be identified }\end{array}$ & $\begin{array}{l}\text { Unenhanced CT: central low attenuation scar } \\
\text { surrounded by well-defined homogeneous } \\
\text { isoattenuating tissue } \\
\text { Arterial phase shows a homogeneous hyperdense } \\
\text { lesion; returns to pre-contrast density during } \\
\text { portal phase that is hypo- or isodense }\end{array}$ & $\begin{array}{l}\text { T1: isointense or slightly hypointense. } \\
\text { Gadolinium produces early enhancement with } \\
\text { central scar enhancement during the delayed } \\
\text { phase } \\
\text { T2: slightly hyperintense or isointense }\end{array}$ \\
\hline $\mathrm{HH}$ & $\begin{array}{l}\text { Hyperechogenic, homogeneous lesion with sharp } \\
\text { outlines }\end{array}$ & $\begin{array}{l}\text { Discontinuous peripheral nodular enhancement in } \\
\text { the arterial phase, with a centripetal fill-in in the } \\
\text { extended portal-venin and later phases }\end{array}$ & $\begin{array}{l}\text { T1: hypointense; discontinuous peripheral } \\
\text { enhancement with centripetal fill-in } \\
\text { T2: hyperintense relative to the spleen }\end{array}$ \\
\hline
\end{tabular}

FNH, focal nodular hyperplasia; HH, hepatic hemangioma; CT, computed tomography. 

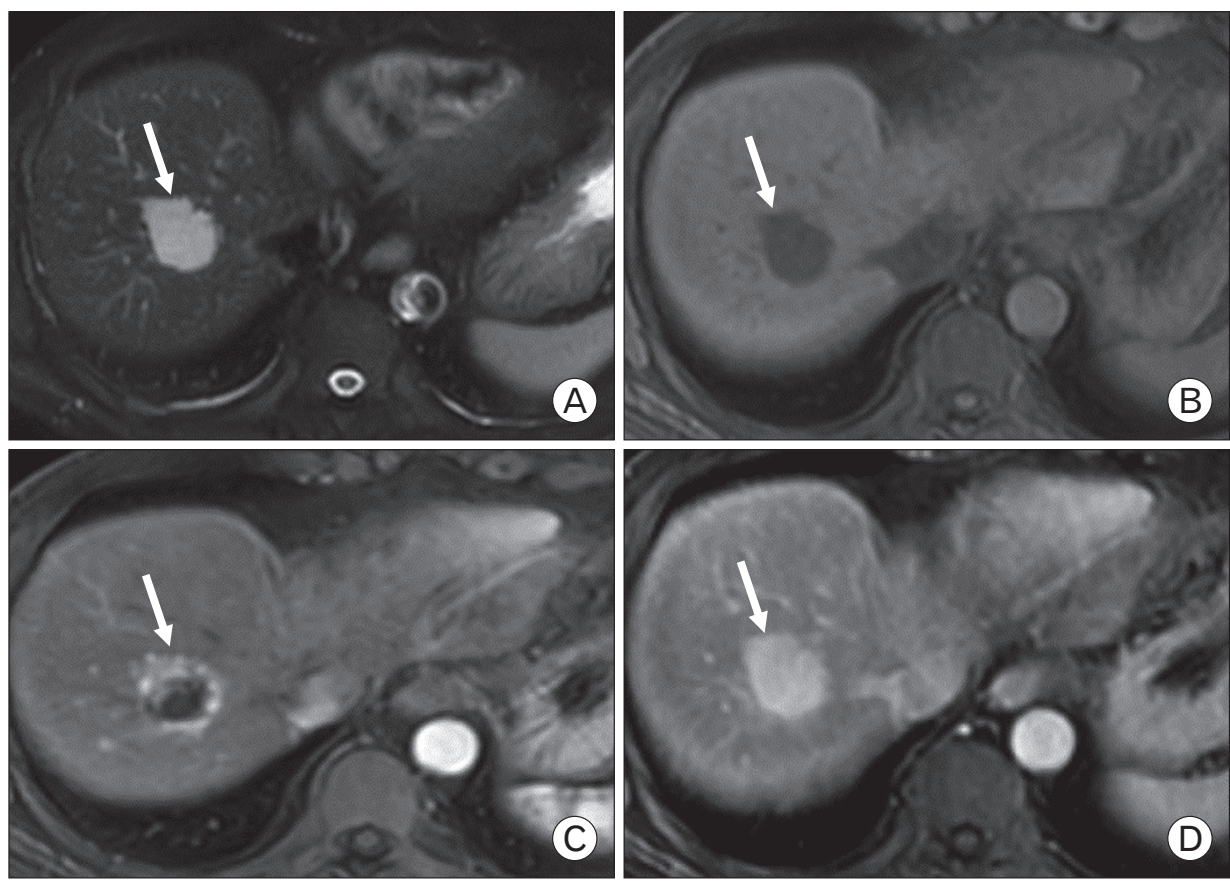

Figure 1. Image depicting typical hepatic hemangioma in a 65-year-old. Axial T2weighted magnetic resonance (MR) image (A) showing a homogeneous hyperintense hepatic lesion (arrow). The lesion was hypointense on axial T1-weighted non-enhanced MR image (B), and shows intense nodular and centripetal progressive enhancement (arrows) on Gadolinium-enhanced MR images (C, D).
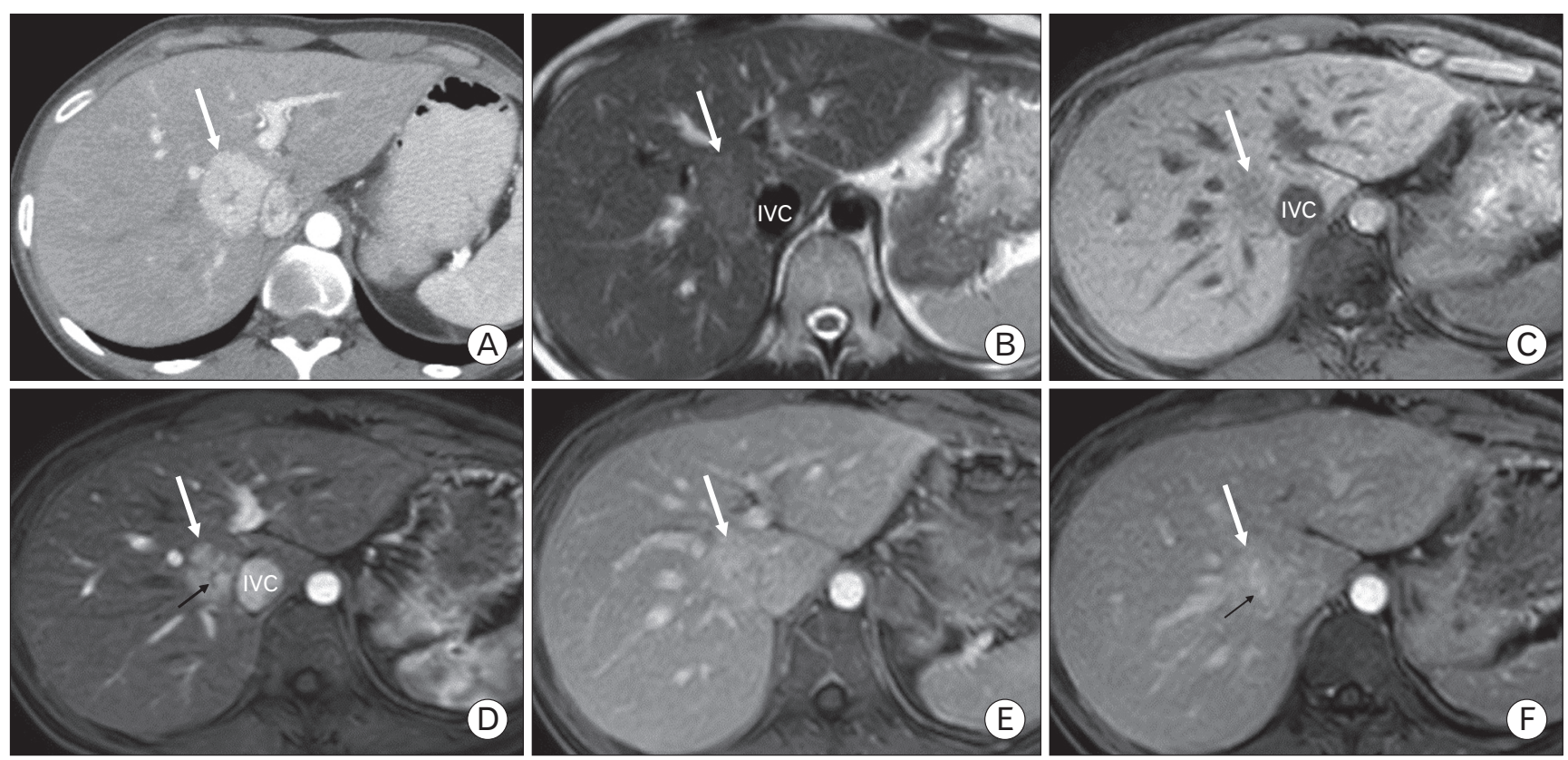

Figure 2. Images depicting contrast-enhanced imaging of a 32-year-old female with focal nodular hyperplasia (FNH), upper abdominal pain, and history of oral contraceptive use. (A) Axial contrast-enhanced computed tomography image showing hypervascular lesion (white arrow) involving segment 4 and 8 of the liver. (B) Axial T2-weighted magnetic resonance (MR) image showing slightly hyperintense lesion (white arrow) located in close proximity to the IVC. (C) Axial non-enhanced T1-weighted MRI image indicating slightly hypointense lesion (white arrow) located in close proximity to the inferior vena cava (IVC). (D) In arterial axial T1-weighted magnetic resonance imaging (MRI) image, the lesion (white arrow) showing high signal intensity than the surrounding liver parenchyma, including small central scar (black arrow), consistent with FNH. (E) Portal phase axial T1-weighted MRI image depicting slightly hyperintense lesion (white arrow) than the surrounding liver parenchyma. (F) Axial T1-weighted MR image obtained in the late venous phase indicating the typical enhanced central scar (black arrow).

management of HHs is generally conservative for typical lesions, in case of a symptomatic or rapidly growing $\mathrm{HH}$, or in the presence of the Kasabach-Merritt syndrome, the patient is referred to a multidisciplinary team (MDT) to discuss treatment options.

\section{FOCAL NODULAR HYPERPLASIA}

FNH is a rare BHT with $0.03 \%$ prevalence estimated by US. ${ }^{13)} \mathrm{FNH}$ are markedly more prevalent in young women, generally detected as sin- 
gle, well-circumscribed, small $(<5 \mathrm{~cm})$ lesions with a central fibrotic scar. When multiple FNH lesions are present, a general vascular hepatic or systemic disorder should be suspected, such as the hereditary hemorrhagic telangiectasia. Moreover, in about $20 \%$ of the cases, FNH may coexist with $\mathrm{HH}$ or HA. ${ }^{14)} \mathrm{FNH}$ is formed as a result of the hyperplastic response to vascular hepatic dystrophy, along with formation of the arteriovenous shunts and the subsequent activation of hepatic stellate cells, which leads to formation of a central scar. ${ }^{15)}$

Further, the role of oral contraceptives and pregnancy in the formation or progression of FNH has not been established yet. ${ }^{16)}$ Most of the FNH cases are asymptomatic, remain stable for prolonged duration, and rarely present with complications. ${ }^{17)} \mathrm{FNH}$ can be diagnosed using contrast-enhanced imaging modalities, such as US, CT, or MRI (Figure 2). The combined use of MRI and hepatobiliary contrast agents, like gadobenate dimeglumine and gadoxetic acid, can help achieve the highest diagnosis accuracy rate, with a specificity and sensitivity of over $90 \%{ }^{18,19)}$ The imaging characteristics of FNH using different imaging modalities have been summarized in Table 1. However, for the exact diagnosis of atypical FNH, a complementary imaging modality is recommended. Additionally, if the imaging modalities fail to establish the diagnosis, a biopsy should be performed to exclude the possibility of HA or HCC.

The management of FNH is generally conservative, and based on the guidelines of the EASL and AASLD, oral contraceptives are not contraindicated in FNH. Moreover, if the diagnosis has been confirmed, then follow-up or surveillance using US is not required. ${ }^{11,12)}$ Additionally, a poor correlation exists between FNH and symptoms, and hence treatment administered is generally inappropriate, even in the symptomatic patients. ${ }^{11)}$ Though surgical resection or partial hepatectomy is the preferred treatment, it is recommended only in special cases, such as the exophytic or pedunculated FNH. Whereas, based on the guidelines of the EASL, non-surgical options, such as the radiofrequency ablation (RFA) and trans-arterial embolization (TAE), are administered to patients unamenable for surgery. ${ }^{11)}$

\section{HEPATIC ADENOMAS}

HAs are rare, benign, epithelial tumors of the liver, observed predomi-

Table 2. Clinical associations and typical MRI findings for hepatic adenoma subtypes

\begin{tabular}{|c|c|c|}
\hline Subtype & Typical MRI signs & Clinical association \\
\hline Inflammatory HA (40\%-55\%) & $\begin{array}{l}\text { Hyperintense on T2-weighted images; persistent enhancement on } \\
\text { delayed phase }\end{array}$ & Metabolic syndrome; obesity; alcohol consumption \\
\hline HNF1A-mutated-HA (30\%-40\%) & Diffuse and homogeneous fat deposition & Adenomatosis; maturity-onset diabetes of the young \\
\hline$\beta$-catenin activated-HA (10\%-15\%) & No typical features & $\begin{array}{l}\text { Androgen use; highest risk for hepatocellular carcinoma; } \\
\text { male gender }\end{array}$ \\
\hline Unclassified HA (5\%-10\%) & No typical features & Unknown \\
\hline
\end{tabular}

MRI, magnetic resonance imaging; HA, hepatic adenoma.
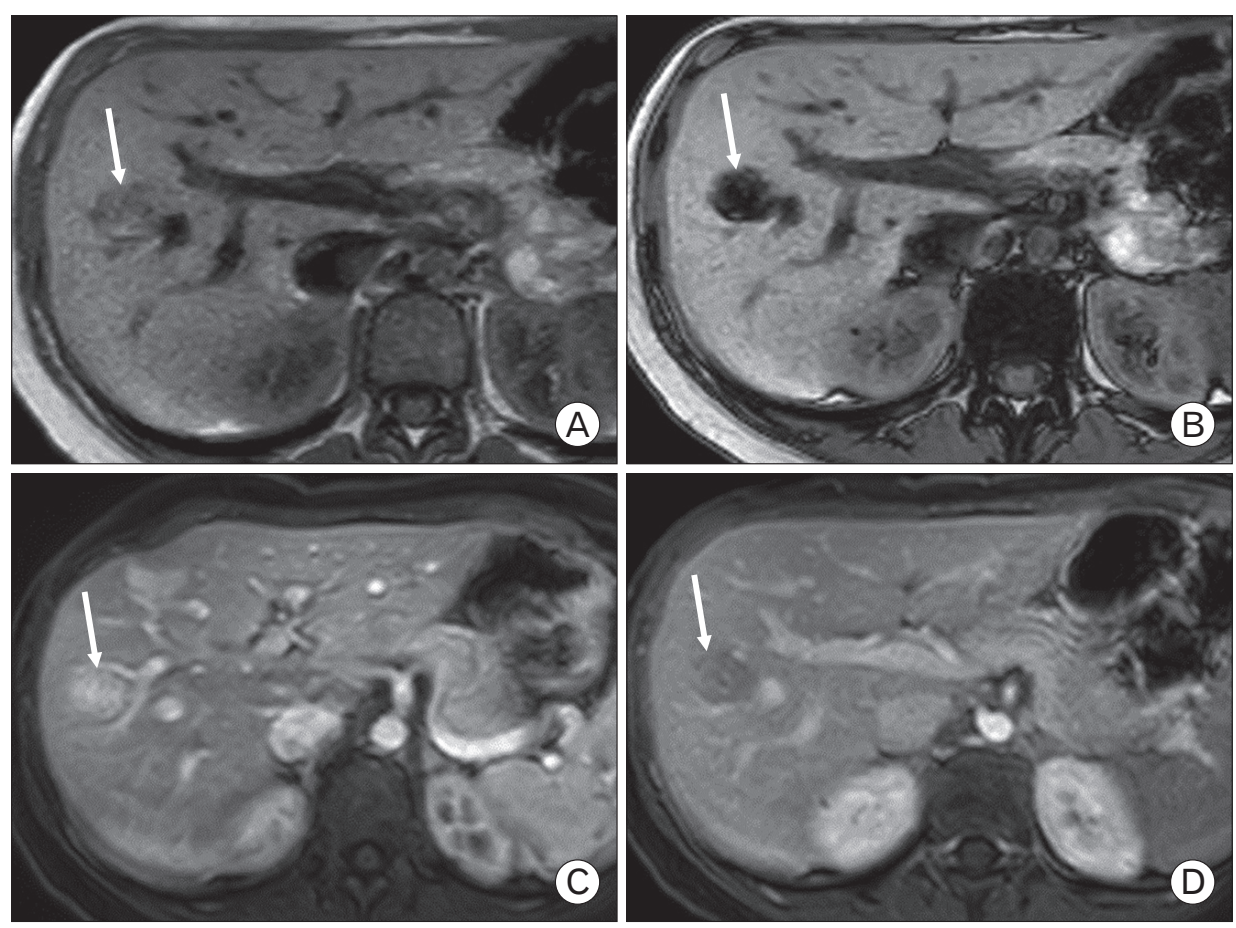

Figure 3. Imaging analysis of a 28-year-old female with hepatic adenoma (HA). T1weighted in- and out-phased magnetic resonance imaging images $(A, B)$, indicating signal drop of the lesion in the segment $\mathrm{V}$ of the liver due to significant presence of intralesion fat. Post dynamic evaluation after Gadolinium administration, the lesion (arrow) showed intense enhancement in the arterial phase $(C)$, with washout in the portal venous phase (D). These imaging features are consistent with the diagnosis of HA. 
nantly in women aged $20-50$ years. ${ }^{20,21)}$ Studies have shown association of use of oral contraceptives as hormonal therapy with incidence of HA in a dose and duration dependent manner. ${ }^{22)}$ Moreover, the incidence of HA is less in the Asian countries possibly due to limited usage of oral contraceptives than in the Europe and North America. ${ }^{23,24)} \mathrm{Ad}-$ ditionally, anabolic androgens, pregnancy, and glycogen storage disease may cause HA. ${ }^{25-27)}$ Moreover, recent data has highlighted the emerging role of obesity and metabolic syndromes as contributing factors for the increased prevalence of HA. ${ }^{28,29)} \mathrm{HAs}$ are usually solitary lesions located in the right lobe of the liver, vary in size from a few millimeters to several centimeters, and rarely pedunculated. The small $(<5$ $\mathrm{cm}$ ) asymptomatic HAs are incidentally diagnosed during imaging for unrelated reasons. However, large HAs may cause mild symptoms of epigastric/right upper quadrant pain, and severe pain may precede bleeding or infarction. Since HAs have been associated with malignant transformation, making distinction between them and other benign liver tumors is thus important. Moreover, rupture and spontaneous hemorrhage are commonly observed in exophytic or large lesions ( $>5$ $\mathrm{cm}){ }^{30)}$

While the risk of malignant transformation to HCC is difficult to ascertain, it is considered rare in small lesions, ${ }^{31)}$ and an increased tumor size in consecutive imaging or a rise in serum alpha-fetoprotein levels may indicate malignant transformation. Additionally, HAs diagnosed in men are significantly associated with higher propensity of malignant transformation. ${ }^{32)}$ Further, multiple genetic alterations have been identified in HAs, which led to the identification of four distinct subtypes: the HNF1A-mutated HA (30\%-40\%), inflammatory HA (40\%$55 \%), \beta$-catenin activated HA (10\%-15\%), and unclassified HA (10\%$25 \%) .{ }^{33)}$ Each individual subtype has different clinical symptoms and varying risk of transformation to HCC (Table 2).

Though multiple imaging techniques aid in the diagnosis, biopsy or surgical resection allow confirmation of HA. The dynamic MRI with a hepatocyte-specific contrast agent is preferred since it allows distinguishing the HA from other benign or malignant lesions in the liver (Figure 3). Moreover, dynamic MRI, with its intrinsic property to detect fat and vascular spaces, offers an opportunity to subtype HA. The MRI characteristics of different subtypes have been summarized in Table 2 . While the hepatocyte nuclear factor- $1 \alpha$ HA or inflammatory HA can be specifically identified using MRI, identification of the $\beta$-catenin activated HA or unclassified HA and their distinction from HCC is not possible using any of the available imaging techniques. Thus, a biopsy should be considered in selected cases.

Further, the patient characteristics, such as gender, tumor size, and progression pattern influence the decisions regarding HA management. While the HA subtypes may also impact disease management, they are yet to be considered due to lack of conclusive evidences. ${ }^{34)}$ As the first therapeutic strategy, patients should be advised for discontinuation of oral contraceptives and anabolic androgens, and management of obesity. Additionally, in case of female patients, a conservative approach has been advised for management of small lesions $(<5 \mathrm{~cm})$ or those that would regress (to $<5 \mathrm{~cm}$ ) following cessation of oral con- traceptives. The EASL guidelines recommend contrast-enhanced MRI at regular intervals of 6-month post-discontinuation of oral contraceptives to determine regression of a large adenoma to $<5 \mathrm{~cm}$. Moreover, imaging using US is a cost-effective strategy, and may be preferred for the easily viewed lesions. Patients that do not show regression of HAs need to undergo treatment, and since men have a higher risk of malignant transformation, adenoma resection is advised irrespective of the size of the lesion upon diagnosis. ${ }^{35)}$ The various approaches for management of HA have been summarized in Figure 4. Furthermore, nonsurgical modalities, such as RFA and TAE, which are minimally invasive and highly successful, may serve as first-line treatments in surgically unamenable patients with spontaneous rupture and bleeding. ${ }^{36}$

However, treatment of HA in pregnant women is challenging, due to the hormone-induced growth, especially in the third trimester, and a possible rupture may result in potentially lethal complications for the mother and fetus. A close and frequent follow-up using US (every 6-12 weeks) is advised to monitor tumor size, and treatment is indicated when the lesion shows signs of growth or bleeding. Moreover, surgery can be safely performed in the first and second trimester ${ }^{37)}$ Though the RFA and TAE are non-invasive alternatives, they are contraindicated during the early phase of pregnancy to minimize complications from radiation or iodinated contrast-exposure to the fetus.

\section{CONCLUSION}

Benign liver tumors are frequently encountered in the clinical practice, and their characterization may be challenging. Thus, obtaining the relevant clinical information, coupled with contrast-enhanced imaging preferably using MRI, facilitates the reliable characterization of most of the lesions in the liver, and subsequently dictates their tailored followup and management. Taken together, an algorithm for the BHT ap-

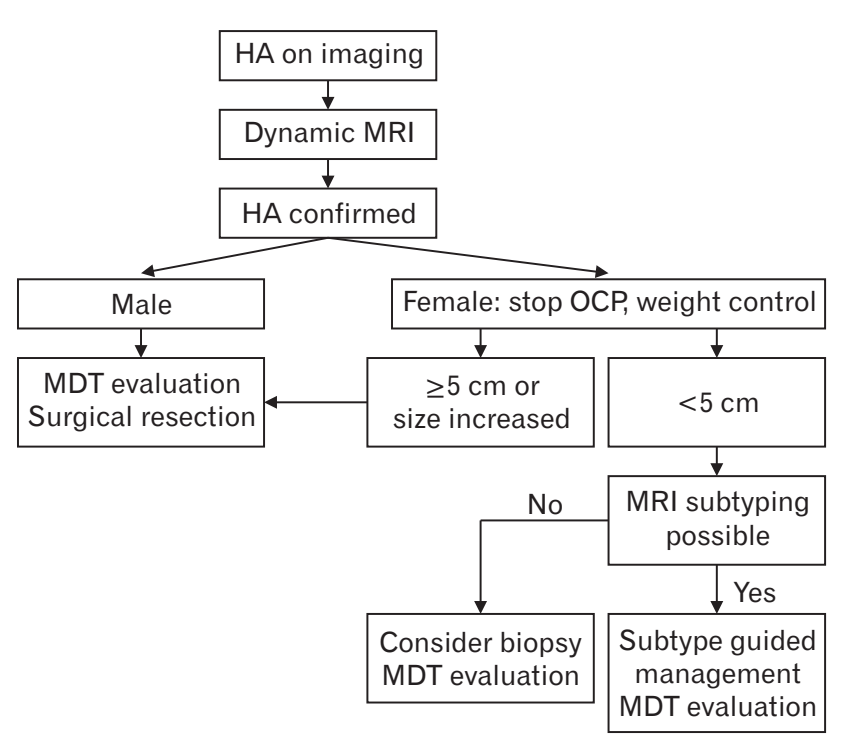

Figure 4. Algorithm for the management of hepatic adenoma (HA). MRI, magnetic resonance imaging; OCP, oral contraceptive pill; MDT, multi-disciplinary team. 


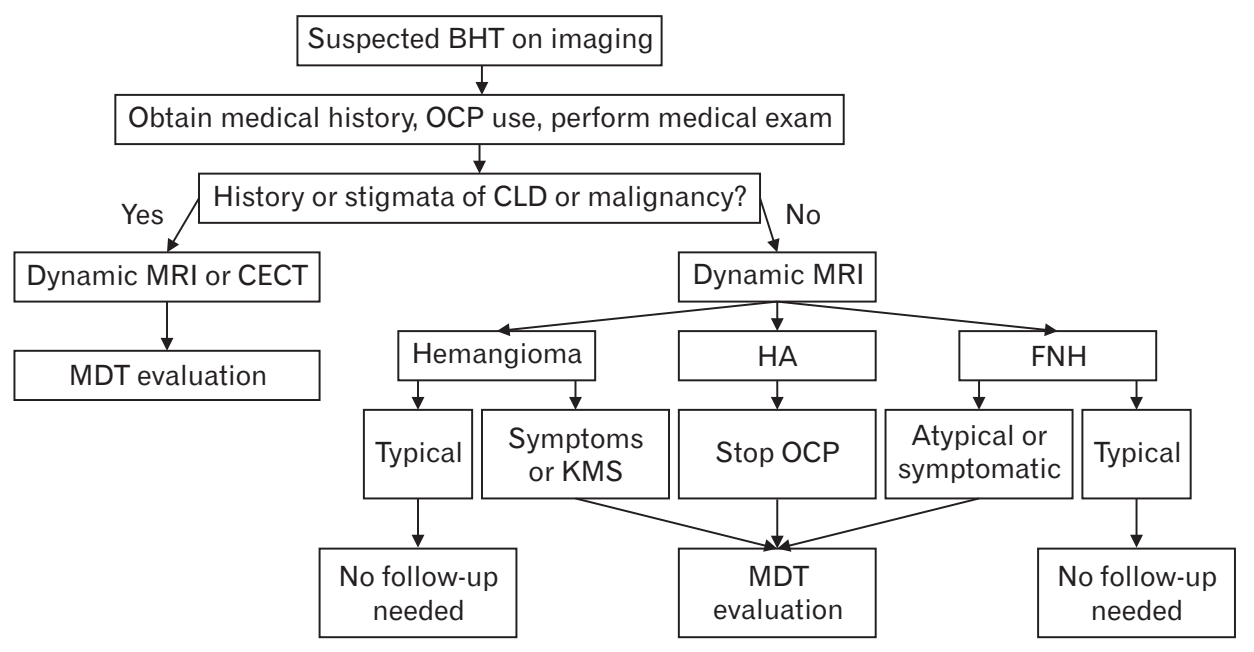

Figure 5. Algorithm indicating approach and management of benign hepatic tumors (BHTs). OCP, oral contraceptive pill; CLD, chronic liver disease; MRI, magnetic resonance imaging; CECT, contrast-enhanced computed tomography; MDT, multi-disciplinary team; HA, hepatic adenoma; FNH, focal nodular hyperplasia; KMS, Kasabach-Merritt syndrome. proach and management, and an MDT approach where possible, has been summarized in Figure 5.

\section{CONFLICT OF INTEREST}

No potential conflict of interest relevant to this article was reported.

\section{ORCID}

Fadi Abu Baker: https://orcid.org/0000-0001-5428-7180

Abdel-Rauf Zeina: https://orcid.org/0000-0002-9224-8657

Saif Abu Mouch: https://orcid.org/0000-0003-2753-4697

Amir Mari: https://orcid.org/0000-0002-6828-4738

\section{REFERENCES}

1. Smith-Bindman R, Miglioretti DL, Johnson E, Lee C, Feigelson HS, Flynn M, et al. Use of diagnostic imaging studies and associated radiation exposure for patients enrolled in large integrated health care systems, 1996-2010. JAMA 2012;307:2400-9.

2. Marin D, Furlan A, Federle MP, Midiri M, Brancatelli G. Imaging approach for evaluation of focal liver lesions. Clin Gastroenterol Hepatol 2009;7:624-34.

3. Rungsinaporn K, Phaisakamas T. Frequency of abnormalities detected by upper abdominal ultrasound. J Med Assoc Thai 2008;91:1072-5.

4. Bahirwani R, Reddy KR. Review article: the evaluation of solitary liver masses. Aliment Pharmacol Ther 2008;28:953-65.

5. Horta G, Lopez M, Dotte A, Cordero J, Chesta C, Castro A, et al. Benign focal liver lesions detected by computed tomography: review of 1,184 examinations. Rev Med Chil 2015;143:197-202.

6. Mergo PJ, Ros PR. Benign lesions of the liver. Radiol Clin North Am 1998;36:319-31.

7. Shaked O, Siegelman ES, Olthoff K, Reddy KR. Biologic and clinical features of benign solid and cystic lesions of the liver. Clin Gastroenterol Hepatol 2011;9:547-62.

8. Gemer O, Moscovici O, Ben-Horin CL, Linov L, Peled R, Segal S. Oral contraceptives and liver hemangioma: a case-control study. Acta Ob- stet Gynecol Scand 2004;83:1199-201.

9. Kasabach HH, Merritt KK. Capillary hemangioma with extensive purpura: report of a case. Am J Dis Child 1940;59:1063-70.

10. Quaia E, Bertolotto M, Dalla Palma L. Characterization of liver hemangiomas with pulse inversion harmonic imaging. Eur Radiol 2002;12: $537-44$.

11. European Association for the Study of the Liver (EASL). EASL clinical practice guidelines on the management of benign liver tumours. J Hepatol 2016;65:386-98.

12. Marrero JA, Ahn J, Rajender Reddy K; American College of Gastroenterology. ACG clinical guideline: the diagnosis and management of focal liver lesions. Am J Gastroenterol 2014;109:1328-48.

13. Toro A, Mahfouz AE, Ardiri A, Malaguarnera M, Malaguarnera G, Loria $\mathrm{F}$, et al. What is changing in indications and treatment of hepatic hemangiomas. A review. Ann Hepatol 2014;13:327-39.

14. Brancatelli G, Federle MP, Grazioli L, Blachar A, Peterson MS, Thaete L. Focal nodular hyperplasia: CT findings with emphasis on multiphasic helical CT in 78 patients. Radiology 2001;219:61-8.

15. Sato Y, Harada K, Ikeda H, Fijii T, Sasaki M, Zen Y, et al. Hepatic stellate cells are activated around central scars of focal nodular hyperplasia of the liver: a potential mechanism of central scar formation. Hum Pathol 2009;40:181-8.

16. Rifai K, Mix H, Krusche S, Potthoff A, Manns MP, Gebel MJ. No evidence of substantial growth progression or complications of large focal nodular hyperplasia during pregnancy. Scand J Gastroenterol 2013;48:88-92.

17. Perrakis A, Demir R, Muller V, Mulsow J, Aydin U, Alibek S, et al. Management of the focal nodular hyperplasia of the liver: evaluation of the surgical treatment comparing with observation only. Am J Surg 2012; 204:689-96.

18. Suh CH, Kim KW, Kim GY, Shin YM, Kim PN, Park SH. The diagnostic value of Gd-EOB-DTPA-MRI for the diagnosis of focal nodular hyperplasia: a systematic review and meta-analysis. Eur Radiol 2015;25:95060.

19. McInnes MD, Hibbert RM, Inacio JR, Schieda N. Focal nodular hyperplasia and hepatocellular adenoma: accuracy of gadoxetic acid-enhanced MR imaging: a systematic review. Radiology 2015;277:413-23. 20. Maillette de Buy Wenniger L, Terpstra V, Beuers U. Focal nodular hy- 
perplasia and hepatic adenoma: epidemiology and pathology. Dig Surg 2010;27:24-31.

21. Lin H, van den Esschert J, Liu C, van Gulik TM. Systematic review of hepatocellular adenoma in China and other regions. J Gastroenterol Hepatol 2011;26:28-35.

22. Rosenberg L. The risk of liver neoplasia in relation to combined oral contraceptive use. Contraception 1991;43:643-52.

23. Rooks JB, Ory HW, Ishak KG, Strauss LT, Greenspan JR, Hill AP, et al. Epidemiology of hepatocellular adenoma: the role of oral contraceptive use. JAMA 1979;242:644-8.

24. Lin H, van den Esschert J, Liu C, van Gulik TM. Systematic review of hepatocellular adenoma in China and other regions. J Gastroenterol Hepatol 2011;26:28-35.

25. Bioulac-Sage P, Sempoux C, Balabaud C. Hepatocellular adenoma: classification, variants and clinical relevance. Semin Diagn Pathol 2017;34:112-25.

26. Labrune P, Trioche P, Duvaltier I, Chevalier P, Odievre M. Hepatocellular adenomas in glycogen storage disease type I and III: a series of 43 patients and review of the literature. J Pediatr Gastroenterol Nutr 1997; 24:276-9.

27. Resnick MB, Kozakewich HP, Perez-Atayde AR. Hepatic adenoma in the pediatric age group. Clinicopathological observations and assessment of cell proliferative activity. Am J Surg Pathol 1995;19:1181-90.

28. Bunchorntavakul C, Bahirwani R, Drazek D, Soulen MC, Siegelman ES, Furth EE, et al. Clinical features and natural history of hepatocellular adenomas: the impact of obesity. Aliment Pharmacol Ther 2011;34: 664-74.
29. Bioulac-Sage P, Taouji S, Possenti L, Balabaud C. Hepatocellular adenoma subtypes: the impact of overweight and obesity. Liver Int 2012; 32:1217-21.

30. Bieze M, Phoa SS, Verheij J, van Lienden KP, van Gulik TM. Risk factors for bleeding in hepatocellular adenoma. Br J Surg 2014;101:847-55.

31. Foster JH, Berman MM. The malignant transformation of liver cell adenomas. Arch Surg 1994;129:712-7.

32. Farges O, Ferreira N, Dokmak S, Belghiti J, Bedossa P, Paradis V. Changing trends in malignant transformation of hepatocellular adenoma. Gut 2011;60:85-9.

33. Bioulac-Sage P, Laumonier H, Couchy G, Le Bail B, Sa Cunha A, Rullier A, et al. Hepatocellular adenoma management and phenotypic classification: the Bordeaux experience. Hepatology 2009;50:481-9.

34. Thomeer MG, Broker M, Verheij J, Doukas M, Terkivatan T, Bijdevaate D, et al. Hepatocellular adenoma: when and how to treat?: update of current evidence. Therap Adv Gastroenterol 2016;9:898-912.

35. Bossen L, Gronbaek H, Lykke Eriksen P, Jepsen P. Men with biopsyconfirmed hepatocellular adenoma have a high risk of progression to hepatocellular carcinoma: a nationwide population-based study. Liver Int 2017;37:1042-6.

36. Van Rosmalen BV, Coelen RJ, Bieze M, van Delden OM, Verheij J, Dejong $\mathrm{CH}$, et al. Systematic review of transarterial embolization for hepatocellular adenomas. Br J Surg 2017;104:823-35.

37. Noels JE, van Aalten SM, van der Windt DJ, Kok NF, de Man RA, Terkivatan T, et al. Management of hepatocellular adenoma during pregnancy. J Hepatol 2011;54:553-8. 